\title{
Opiniones de estudiantes discapacitados sobre la utilización del ordenador en la educación a distancia: Resultados de un estudio empírico
}

\author{
(Using computers in distance study: \\ results of a survey amongst disabled distance students)
}

\author{
Rainer Ommerborn \& Rudolf Schuemer \\ FernUniversität (Alemania)
}

\begin{abstract}
RESUMEN: Existe el riesgo de que la euforia reinante sobre la aplicación de los nuevos medios en el ámbito educativo impida ver que su creciente aplicación y la progresiva "virtualización" puede provocar además la marginación de determinados grupos de personas interesadas en la educación. En este estudio algunos estudiantes a distancia discapacitados fueron preguntados sobre su experiencia en el uso del PC e Internet. Podemos adelantar el resultado principal: por regla general, los consultados ven muchas más ventajas que inconvenientes en su utilización, aunque observan algunos riesgos. El siguiente informe pretende contribuir a sensibilizar a los responsables de las instituciones educativas sobre las especiales necesidades de los estudiantes discapacitados.
\end{abstract}

\section{Virtualización - estudiantes discapacitados - necesidades especiales}

ABSTRACT: In the widespread euphoria about the new technologies in distance education we run the danger of not sufficiently considering how the ever increasing "virtualisation" may exclude some student groups. We therefore undertook an explorative survey asking disabled students about their experiences with using computers and the Internet. To name the main result first: all in all, those questioned do indeed mention more advantages than disadvantages; they do, however, also mention dangers. The following article is addressed to those in a position of responsibility in educational institutions in the hope of sensitising them to the special needs of disabled students.

Virtualisation - disabled students - special needs 


\section{PLANTEAMIENTO}

Los «nuevos medios» $»^{1}$ en particular Internet, han adquirido en los últimos años una gran importancia en la enseñanza y el aprendizaje en la universidad ${ }^{2}$ y en particular en la enseñanza a distancia. Las instituciones de enseñanza a distancia de todo el mundo están realizando un gran esfuerzo para poner estos nuevos medios al servicio de sus objetivos ${ }^{3}$. Esta tendencia a la implantación de los nuevos medios se manifiesta en el aumento de los trabajos sobre el tema «Internet» 0 «multimedia» publicados en los últimos años en las revistas especializadas en educación a distancia, como Distance Education, Epistolodidaktika u Open Learning.

La FernUniversität, que intenta aunar sus esfuerzos en este sentido bajo el título programático «universidad virtual», ofrece cada vez más productos multimedia, en soporte CD-ROM o disponibles a través de Internet.

Los nuevos medios ofrecen grandes posibilidades ${ }^{5}$ precisamente a los estudiantes discapacitados, aunque por otro lado entrañan también - al menos para al gunos de ellos - el riesgo de un mayor aislamiento. Muchos de los elementos de uso y funciones del PC, como la navegación con el ratón o la utilización de combinaciones de teclas ('short cuts'), sólo pueden ser utilizados por algunos discapacitados (como los que tienen limitada la motricidad de los brazos) con un gran esfuerzo o incluso dolor. Las interfaces gráficas del usuario, tan habituales hoy en día, pueden plantear grandes dificultades a los discapacitados visuales. Por otro lado, los «nuevos medios» ofrecen a los estudiantes discapacitados muchas posibilidades de compensación de los déficits derivados de su discapacidad y de participación en la vida académica: incluso los discapacitados físicos con severas limitaciones de movilidad pueden participar directamente a través de Internet en debates y conferencias («seminarios virtuales»).

EI presente estudio pretende, a través de una encuesta exploratoria, recoger las opiniones de los implicados respecto a las oportunidades y riesgos de los nuevos medios.

\footnotetext{
es decir, tecnologías de la información y la comunicación basadas en la informática (incl. Internet y "multimedia"; sobre el concepto de multimedia vid. e.o. Schulmeister 1997, Cap. 2).

2 vid. Ios últimos números de la serie "Die Medien in der Wissenschaft"- e.o.: Hauff 1998; Krahn \& Wedekind 2000; Scheuermann 2000; Simon 1997.

vid. e.o. las últimas conferencias del International Council for Distance Education, ICDE, 1997 y 1999, así como Daniel 1996; Holmberg 1996; Fandl, Bartz \& Nickolmann 1996.

${ }^{4}$ vid. p.ej. B. Schlageter, Buhrmann \& Mittrach 1997; vid. además las páginas web de la FernUniversität: http://www.fernuni-hagen.de

${ }^{5}$ La importancia de estos medios en la integración de personas discapacitadas se manifiesta en los trabajos publicados en revistas especializadas (vid. Heiler 1997), en Internet (vid. Zondanella 1998; vom Bruch 1998a) y en las declaraciones de las asociaciones de discapacitados (vid. vom Bruch 1998b).
} 
Opiniones de estudiantes discapacitados sobre la utilización del ordenador en la educación a distancia

\section{SOBRE EL GRUPO DE ESTUDIANTES DISGAPACITADOS EN LA EDUCACIÓN A DISTANCIA}

\section{LOS DISCAPACITADOS COMO GRUPO DESTINATARIO DE LA FERNUNIVERSITÄT}

Entre las funciones de la FernUniversität ${ }^{6}$ figura el ofrecer la oportunidad de recibir formación y la posibilidad de estudiar a aquellos grupos que sufren limitaciones temporales y locales, y no tienen o son muy escasas sus posibilidades de acogerse a la oferta educativa de las universidades presenciales tradicionales. A estos grupos pertenecen las personas que trabajan, las familias monoparentales que tienen a su cargo menores o familiares necesitados de cuidados, y además los discapacitados y enfermos crónicos. Para las personas que desean estudiar y cuya discapacidad o enfermedad limita su movilidad, la educación a distancia constituye una alternativa válida a los estudios presenciales ${ }^{7}$. Y mucho más te niendo en cuenta que en muchas universidades presenciales las condiciones de estudio para los discapacitados no son precisamente las ideales ${ }^{8}$, aunque hay que reconocer los avances habidos en los últimos años, al menos en algunos sectores (siguiendo las recomendaciones de las conferencias de ministros de Educación o de rectores de universidad) ${ }^{9}$.

\section{SOBRE EL CONGEPTO DE DISCAPACITADO}

Parece ser que no existe una definición plenamente aceptada de «discapacidad», pues suele darse en función de determinados objetivos y/o determinadas perspectivas concretas ${ }^{10}$. Sirvan como ejemplo tan solo tres definiciones:

«Discapacitado es aquél cuya capacidad de rendimiento físico y/o psíquico difiere negativamente de la norma, representada por la persona plenamente capaz.» $»^{11}$

«Discapaci dad (disability) designa las consecuencias perjudiciales personales y sociales de una deficiencia (impairment). La deficiencia es toda divergencia de un individuo respecto de las normas (ideales, medias) funcionales o físicas. El perjuicio (handicap) designa las dificultades a las que un discapacitado debe

${ }^{6}$ vid. Peters 1976; Rau 1976; vid. además Kramme 1996

' vid. e.o.: H ofmann \& Ommerborn 1985; Hofmann \& Ommerborn 1997, pág. 33 y ss.; Ommerborn 1995; vid. además Holmberg 1995, pág. 13

${ }^{8}$ vid. e.o. Hofmann \& Ommerborn 1997, pág. 25; vid. además M eister 1998, pág. 2 y ss. y pág. 149 y SS.

9 Deutsches Studentenwerk 1993

${ }^{10}$ vid. e.o. Adam 1993, pág. 24

"Kranig \& Ramm 1990, pág. 4 
enfrentarse en su entorno. La deficiencia por el contrario se refiere a la enfermedad en situación irreversible (p.ej. malformación de miembros frente a rotura de huesos). Estas definiciones son limitaciones típicas ideales. $\gg^{12}$

«Discapacidad: limitación física, psíquica o mental de carácter permanente que provoca un grado de minusvalía $\mathrm{mMdE}^{*}$ del $10 \%$, con independencia de si es de nacimi ento o es consecuencia de un agente externo (p.ej. accidente, guerra) o enfermedad. Existe discapacidad grave según lo dispuesto sobre protección y ayuda en la Ley de discapacitados graves (SchwbG) en su edición de 8.10.1979 (pág. 1649 del boletín alemán BGBI. I), cuando la disminución de la capacidad productiva para el trabajo es permanente, y del 50\% como mínimo». ${ }^{13}$

En este tipo de descripción destaca la referencia a una norma siempre determinada ${ }^{14}$, siendo la divergencia de esta «norma», la situación o capacidad funcional inferior a la normal, la característica constitutiva del concepto «discapacidad». No podemos entrar ahora a discutir en detalle la problemática de estas definiciones ${ }^{15}$, aunque destacaremos algunos aspectos:

- La referencia a una norma plantea entre otras la cuestión de quién define dicha norma, con qué objetivo y según qué puntos de vista y criterios.

- La definición de discapacidad como divergencia de la norma está ligada a determinadas circunstancias temporales y socioculturales ${ }^{16}$, pues no en vano las normas generalmente exigibles se imponen como consecuencia de procesos sociales y políticos en una situación histórica concreta.

D Al designar un grupo de personas como «fuera de lo normal» se plantea el riesgo de su estigmatización discriminatoria, con todas sus consecuencias negativas. Esto ocurre incluso con la legislación social, donde el concepto de discapacidad (y los perjuicios derivados para los afectados) fundamenta el derecho a medidas y prestaciones compensatorias.

- La clasificación en categorías como «discapacitado» o incluso subcategorías como «discapacitado físico» no pueden hacernos olvidar las considerables diferencias entre los individuos, el hecho de que la discapacidad representa siempre una situación muy particular. Los discapacitados se diferencian entre sí tanto como los no discapacitados. Por el contrario,

${ }_{12}$ Diccionario psicológico Dorsch, 11. edición revisada: Dorsch, Häcker \& Stapf 1987, pág. 86; vid. también la definición en "Klinischen Wörterbuch" (Pschyrembel 1990, pág. 190), que destaca la "disminución de la capacidad productiva para el trabajo "(MdE).

${ }^{13}$ en Pschyrembel 1990, pág. 190; figura: “MdE: Disminución de la capacidad productiva para el trabajo, por accidente o enfermedad; Concepto del derecho de seguros “; pág. 1033

${ }^{14}$ referencia explícita o referencia indirecta o implícita como en Pschyrembel, en la que la gravedad de la discapacidad se expresa con el porcentaje de la capacidad laboral total.

${ }^{15}$ para más detalles, vid. e.o. Adam 1993, pág. 24 y ss. u Ommerborn 1994, pág. 43 y ss. y 139 y ss.; vid. además Münch 1997.

${ }^{16}$ vid. e.o. Müller 1996 
Opiniones de estudiantes discapacitados sobre la utilización del ordenador en la educación a distancia

discapacitados y no discapacitados comparten muchas más coincidencias que diferencias ${ }^{17}$.

Da valoración ajena y la propia de una discapacidad no tienen que coincidir: No todo el considerado «discapacitado» o «discapacitado grave» en sentido jurídico social, se considera a sí mismo discapacitado o perjudicado en aquellos aspectos de la vida que le son importantes. La discapacidad o enfermedad crónica de un estudiante no tiene que provocar necesariamente un perjuicio en sus estudios (hablaremos más adelante sobre ello).

A pesar de lo problemático que resulte el concepto de discapacitado, parece lógico examinar las opiniones de las personas que se consideran a sí mismos discapacitados/enfermos crónicos, aunque sin olvidar la problemática expuesta sobre estos conceptos.

\section{SOBRE LA CIFRA DE ESTUDIANTES DISCAPACITADOS}

Las estimaciones sobre el porcentaje de discapacitados y enfermos crónicos entre los estudiantes se suelen basar en los resultados de las encuestas realizadas entre los propios estudiantes, sobre si padecen discapacidades o enfermedades crónicas y en caso afirmativo sobre el grado de prejuicio que ello ocasiona en sus estudios. En la mayoría de los casos no se ofrece una definición explícita de «discapacidad» o «enfermedad crónica»; la definición se obtiene más bien a posteriori, sobre la base de las declaraciones de los afectados.

De acuerdo con el procedimiento esbozado, a los encuestados en el Estudio Social realizado a nivel nacional por la organización Deutsche Studentenwerk (DSW) se les preguntó en primer lugar: «¿Sufre alguna discapacidad o enfermedad crónica/de larga duración?»; a los que contestaban afirmativamente se les preguntaba a continuación: «¿De qué tipo es su problema de salud?», siendo las respuestas muy variadas ${ }^{18}$. Según el 14을 Estudio Social del año 1994, el 2,3 \% de Ios encuestados se consideraban discapacitados y un 10,4\% enfermos crónicos ${ }^{19}$. De este total de $12,7 \%$ entre discapacitados y enfermos crónicos, no todos se sentían perjudicados por la discapacidad o la enfermedad crónica (vid. Tabla 1; las encuestas entre estudiantes discapacitados de la FernUniversität ${ }^{20}$ arrojan resultados similares).

${ }^{17}$ vid. al respecto p.ej. Dickopp 1983, pág. 353

${ }^{18}$ vid. Schnitzer, Isserstedt, Schreiber \& Schröder 1996; vid. el tipo de estudios análogo en el 13ạ Estudio Social del DSW (Schnitzer, Isserstedt, Kahle, Leszczensky \& Schreiber 1992).

${ }_{19}$ vid. Schnitzer, Isserstedt, Schreiber \& Schröder 1996, cuadro 13.2, pág. 29

${ }^{20}$ Hofmann 1989 
Tabla 1: Grado de perivicio en los estudios motivado por la discapacidad o la enfermedad crónica (según el $14^{\circ}$ Estudio Social de la DSW)

\begin{tabular}{l|r}
\hline GRADO DE PERJUICIO: & $\%$ \\
\hline no perjudicado en sus estudios en absoluto & 50 \\
\hline algo perjudicado en sus estudios & 26 \\
\hline medianamente perjudicado en sus estudios & 16 \\
\hline muy perjudicado en sus estudios & 8 \\
\hline
\end{tabular}

Una cuarta parte (24\%) de los estudiantes discapacitados y enfermos crónicos se siente perjudicado en sus estudios «medianamente» 0 «mucho». Con respecto al total de estudiantes, aproximadamente el $3 \%$ se sienten perjudicados en sus estudios debido a su discapacidad o su enfermedad crónica. Los anteriores estudios sociales arrojaron cifras similares. En virtud de los datos del 13 - Estudio Social, la DSW estimó «que hay más de 200.000 estudiantes con enfermedades crónicas o discapacidades, de los cuales más de 50.000 se si enten medianamente o muy perjudicados ${ }^{21} \gg$.

La FernUniversität no dispone de datos estadísticos actualizados sobre el porcentaje de estudiantes discapacitados, pues desde 1988 y en aplicación de la normativa sobre protección de datos la característica «gravemente discapacitado: sí/no» ya no figura en los formularios de matriculación ${ }^{22}$. Los estudios anteriores permiten estimar que sobre un $2 \%$ de nuestros estudiantes se consideran a sí mismos discapacitados ${ }^{23}$. Estimaciones similares se derivan de estudios posteriores, lo que para el año 1994 supone un número absoluto de unos 1.100 matriculados discapacitados en la FernUniversität. ${ }^{24}$

En los estudios de la DSW el porcentaje de estudiantes discapacitados en la FernUniversität es inferior al de la universidad presencial: esto puede resultar paradójico, pues los discapacitados son uno de los grupos de destinatarios explícitos de la FernUniversität, para facilitarles el acceso a los estudios superiores. Pero hay que tener en cuenta las diferencias en el método: en los estudios de la DSW se preguntaba de forma explícita por discapacidad y enfermedad crónica, mientras que en la FernUniversität hasta 1988 sólo figuraba la característica «discapacitado grave»

${ }^{21}$ DSW 1993, pág. 2

${ }^{22}$ desde 1988 la "Comisión especial para estudiantes discapacitados" les ofrece la posibilidad de registrarse (de forma voluntaria). - Hay que destacar, que la petición de la Comisión especial se dirige a "estudiantes discapacitados " (y no como en las encuestas de la DSW también a los "enfermos crónicos ").

${ }^{23}$ H ofmann \& Ommerborn 1986, pág. 199. Es probable que estas estimaciones supongan una infravaloración, pues hay estudiantes discapacitados que por temor a la estigmatización prefieren no registrarse (vid. Ommerborn 1994, pág. 136).

${ }^{24}$ según Hofmann 1994, pág. 2, para el semestre de verano de 1994 
Opiniones de estudiantes discapacitados sobre la utilización del ordenador en la educación a distancia

en el formulario de matriculación y en los estudios posteriores sólo se preguntaba por la discapacidad, pero no de forma explícita por las enfermedades crónicas.

\section{SUBGRUPOS DE DISCAPACITADOS Y ENFERMOS CRÓNICOS / SISTEMA DE CATEGORÍAS}

El establecimiento de subgrupos o categorías de discapacidad suele realizarse desde puntos de vista pragmáticos; la enorme variedad de formas de discapacidad/enfermedad crónica debe encuadrarse en un número limitado de grupos/categorías. Estas categorías permiten desarrollar ayudas específicas para cada grupo: por ejemplo, las personas con graves deficiencias visuales precisan ayudas protésicas diferentes de las que precisan los usuarios de sillas de ruedas. Las ventajas e inconvenientes de los diferentes sistemas de clasificación sobre discapacidades y enfermedades crónicas no deben cuestionarse aquí; aunque cabe señalar que incluso los «problemas en los estudios derivados de discapacidades y enfermedades crónicas» admiten diversos esquemas, como se observa al comparar las categorías en los diferentes estudios. ${ }^{25}$

Estas categorías no se suelen concebir como excluyentes para que el encuestado se incluya sólo en una. Como muchos afectados no sufren una única forma de discapacidad, sino una discapacidad múltiple, se prevé de forma expresa la posibilidad de citar varias categorías. Estos sistemas por regla general no pueden considerarse completos, pues no incluyen una categoría residual (como «otra forma de discapacidad»).

Señalaremos aquí dos sistemas. En primer lugar, las categorías del 14을 Estudio Social de la DSW ${ }^{26}$ : (1) Alergias / enfermedades respiratorias; (2) deficiencias del aparato locomotor; (3) deficiencias visuales; (4) enfermedades de los órganos internos / trastornos del metabolismo; (5) enfermedades psíquicas; (6) enfermedades de la piel; (7) deficiencias de garganta, nariz u oído; (8) deficiencias del sistema nervioso central; (9) otras deficiencias. Las categorías en el estudio de la FernUniversität ${ }^{27}$ eran las siguientes: (1) usuario de silla de ruedas; (2) discapacitado físico de otro tipo; (3) sufre deficiencias en los órganos internos; (4) ciego; (5) sufre deficiencia visual severa; (6) sordo; (7) sufre deficiencia auditiva severa; (8) discapacitado psíquico; (9) otros.

El sistema de categorías utilizado en este estudio (vid. Tabla 2) mezcla ambas clasificaciones, permitiendo de esta manera la comparación los estudios DSW y con anteriores estudios de la FernUniversität.

\footnotetext{
${ }^{25}$ como en DSW (vid. e.o. Schnitzler et al 1996), en estudios de la FernUniversität (H ofmann 1989), la Open University británica (Child 1993) o la Open universiteit holandesa (1992).

${ }^{26}$ Schnitzer et al 1996

27 Hofmann 1989
} 
3. MÉTODO

En otoño de 1999 se realizó una encuesta escrita mediante un cuestionario parcialmente estandarizado. (El cuestionario se desarrolló previa consulta con grupos de estudiantes discapacitados y una encuesta previa). Se envió a 300 estudiantes, que se habían registrado como discapacitados en la Comisión especial para estudiantes discapacitados de la FernUniversität. 105 de ellos devolvieron el cuestionario cumplimentado, lo que supone una cuota de devolución del 35\%. Cuotas similares son las obtenidas en otras encuestas de estudiantes a distancia, como por ejemplo en un estudio sobre «dotación de estudiantes a distancia con ordenadores» durante el curso 1995/96 ${ }^{28}$.

La representatividad de los resultados de la encuesta es cuestionable debido entre otros al método de selección y la escasa cuota de devolución (posible 'non-response bias'). Esta falta de representatividad tampoco nos parece tan relevante, dado que la encuesta no pretendía llegar a resultados representativos, sino más bien hacer una primera indagación sobre el estado de opinión de los afectados, conocer los problemas y obtener posibles sugerencias y propuestas de solución. En cualquier caso, dado el escaso número de respuestas en los cuestionarios devueltos, los resultados sobre subgrupos deben interpretarse como tendencias. ${ }^{29}$

\section{RESULTADOS}

\section{CARACTERÍSTICAS DE LOS ENCUESTADOS Y DATOS SOBRE EL ESTUDIO}

Edad, sexo y lugar de residencia de los encuestados: Los encuestados tenían una edad media de 43 (mediana: 40 años), superior a la de los estudiantes de la FernUniversität en el semestre de invierno 98/99 en total (mediana: 32 años). EI porcentaje de mujeres era del $43 \%$, algo superior al de los estudiantes a distancia en total (37\%). Alrededor de un tercio de los encuestados vive en Renania del Norte - Westfalia.

Tipo de discapacidades ${ }^{30}$ : Un 45\% de los encuestados indican más de una forma de discapacidad o enfermedad, son discapacitados múltiples. Las deficiencias «del aparato locomotor» son las más habituales (38\% del total), seguidas de las «del sistema nervioso central» y «de los órganos internos /enfermedades metabó-

${ }^{28}$ von Prümmer \& Rossié 1996, pág. 1

${ }^{29}$ más detalles sobre le método vid. Ommerborn \& Schuemer 2000.

${ }^{30}$ Muchos de los encuestados no señalaron con una cruz una o más de las categorías sugeridas, sino que ampliaron datos sobre su discapacidad, que se añadieron a las categorías presentadas (en la medida de lo posible según el esquema de Budde \& Leszczensky 1990). 
Opiniones de estudiantes discapacitados sobre la utilización del ordenador en la educación a distancia

licas crónicas» (un 12,7\% respectivamente); «deficiencias visuales» en un 9,8\%, y «deficiencias auditivas» en un 8,7\% y «discapacidades psíquicas» en un 9,2\% del total (vid. Tabla 2). Los porcentajes fueron similares en un estudio anterior en la FernUniversität ${ }^{31}$, pero muy distintos en los estudios sociales de la DSW: mientras que en éste se citan más «alergias/ enfermedades respiratorias» (del $51 \%$ de los encuestados; $36 \%$ de todas las respuestas), la misma categoría en este estudio fue señalada sólo por un $9 \%$ de los encuestados (aprox. 5\% de las respuestas). ${ }^{32}$

Tabla 2: Frecuencia de las formas de discapacidad (Posibilidad de selecciones múltiples)

Datos del estudio en la FernUniversität. $\mathrm{N}=104$ (un encuestado no respondió la pregunta 4 en total). f: Frecuencia; \%B: porcentaje, referido a 104 encuestados que responden; \% $\mathrm{Ngl}$ : Porcentaje, referido al total de 226 respuestas - sin las subcategorías de deficiencias del aparato locomotor; \%Ng2: Porcentaje, referido al total de 173 respuestas - con las subcategorías de deficiencias del aparato locomotor.

discapacidad física/deficiencia del aparato locomotor: indicación de una o varias de las cuatro categorías siguientes (o combinación).

- deficiencia del aparato locomotor en total

- deficiencias del aparato locomotor con limitaciones en la motricidad de la parte superior del cuerpo, en particular de brazos y manos

- deficiencias del aparato locomotor con limitaciones en la motricidad de las piernas.

\begin{tabular}{|r|r|r|r} 
& & & \\
\hline 66 & 63,5 & 15,0 & 38,2 \\
\hline 34 & 32,7 & 15,0 & - \\
\hline & & & \\
\hline 24 & 23,1 & 10,6 & - \\
\hline 36 & 34,6 & 15,9 & - \\
\hline 25 & 24,0 & 11,1 & - \\
\hline 22 & 21,2 & 9,7 & 12,7 \\
\hline 22 & 21,2 & 9,7 & 12,7 \\
\hline 9 & 8,7 & 4,0 & 5,2 \\
\hline 5 & 4,8 & 2,2 & 2,9 \\
\hline 17 & 16,3 & 7,5 & 9,8 \\
\hline 15 & 14,4 & 6,6 & 8,7 \\
\hline 16 & 15,4 & 7,1 & 9,2 \\
\hline 1 & 1,0 & 0,4 & 0,6 \\
\hline
\end{tabular}

- usuario de silla de ruedas

deficiencias del sistema nervioso central

deficiencias en los órganos internos / enfermedades metabólicas crónicas.

\begin{tabular}{l|r|r|r|r}
\hline alergias crónicas / enfermedades respiratorias ............... & 9 & 8,7 & 4,0 & 5,2 \\
\hline enfermedades crónicas de la piel..................................... & 5 & 4,8 & 2,2 & 2,9 \\
\hline deficiencias visuales ....................................................... & 17 & 16,3 & 7,5 & 9,8 \\
\hline deficiencias auditivas / discapacidades auditivas ............. & 15 & 14,4 & 6,6 & 8,7 \\
\hline discapacidades psíquicas .............................................. & 16 & 15,4 & 7,1 & 9,2 \\
\hline otras discapacidades (sin mayor especificación) ............... & 1 & 1,0 & 0,4 & 0,6 \\
\hline
\end{tabular}

${ }^{31}$ Hofmann 1989

${ }^{32}$ Sobre las posibles causas de estas discrepancias vid. Ommerborn \& Schuemer 2000, pág. 28 y s. 
Tipo de asistencia: Alrededor de $2 / 3$ de los encuestados son estudiantes a tiempo parcial y un 1/5 a tiempo completo; el $12 \%$ están matriculados como oyentes. Esta distribución en función del tipo de asistencia de los encuestados corresponde a la tendencia general de los estudiantes a distancia; hay mayor diferencia en comparación con el total de los estudiantes a distancia, sobre todo en los que cursan segundos estudios, que entre los encuestados es de un $1 \%$ frente al $11 \%$ del total de estudiantes.

Elección de especialidad / Materias de estudio: Mientras que más de la mitad del total de los estudiantes de la FernUniversität se matriculan en «Ciencias económicas» (WIWI) y alrededor de un 1/4 en «Educación, ciencias sociales, psicología y humanidades» (ESGW), esta relación se invierte entre los encuestados: $58 \%$ por ESGW y $28 \%$ por WIWI; «Psicología» y «Ciencias de la conducta social» son las más escogidas ( $17 \%$ del total). En el estudio social de la Deutsches Studentenwerk se observa también la preferencia de filologías y ciencias culturales, así como de las especialidades de las ciencias sociales entre los estudiantes discapacitados / enfermos crónicos.

Número de semestres y abandono de los estudios: Aprox. la mitad de los encuestados ha estudiado 50 más semestres y alrededor del 1/4 ha estudiado 90 más semestres en la FernUniversität. Por lo que respecta al abandono de los estudios, los encuestados señalan mayoritariamente «en el ciclo básico» con un 58\%; alrededor del $23 \%$ lo dejan «en el ciclo principal» y aprox. el $13 \%$ «poco antes de la licenciatura»; algunos ya han terminado una licenciatura en la FernUniversität y estudian para una segunda o se preparan para un postgrado.

Asistencia a los centros de estudio: La FernUniversität dispone de una amplia red de centros de estudio distribuida por toda Alemania, pero especialmente densa en Renania del Norte-Westfalia. Durante el último semestre anterior a la encuesta más de la mitad de los encuestados (54\%) no había asistido a ningún centro de estudio; tan sólo una minoría (11\%) había asistido una vez por semana o más al centro de estudio. El porcentaje de los no asisten a estos centros es muy superior entre los usuarios de sillas de ruedas, dado que algunos no disponen de accesos apropiados.

\section{UTILIZACIÓN DEL PC: VENTAJAS E INCONVENIENTES}

Utilización del PC: Con un 30\%, el número de estudiantes discapacitados encuestados que no utilizan un PC en sus estudios es claramente superior al del total de los estudiantes de la FernUniversität (alrededor del 14\% según un estudio ${ }^{33}$ del curso 1995/96). Por subgrupos en función del tipo de discapacidad/ enfermedad, no se aprecian diferencias a nivel global respecto al uso del PC,

${ }^{33}$ von Prümmer \& Rossié 1996 
Opiniones de estudiantes discapacitados sobre la utilización del ordenador en la educación a distancia

aunque el porcentaje de no usuarios es bastante superior entre los encuestados con deficiencias visuales o discapacidades psíquicas (más de la mitad en cada caso).

Motivos para la no utilización de un PC: El motivo más citado se refiere al elevado coste de adquisición y funcionamiento. Pero también se cita «el temor a que el manejo del ordenador requiere mucho tiempo y esfuerzo, y no merece la pena» así como el coste y las escasas posibilidades de aprendizaje. EI «temor a que su discapacidad en concreto limite su manejo» se cita poco en comparación con otros motivos, excepto los deficientes visuales que lo citan mayoritariamente, además de otras causas específicas de su discapacidad: «El temor de sobreesfuerzo de la vista por la utilización del PC» así como la «preferencia por un aparato de lectura independiente del PC», que aún con una visión muy escasa, resulta más cómodo que la pantalla del monitor, incluso aumentándola.

Utilización de accesorios especiales para discapacitados: Son pocos los encuestados que utilizan equipos especiales para discapacitados, debido a su alto coste y la escasa información disponible: muchos encuestados desearían que la FernUniversität ofreciera más información al respecto.

Ventajas einconvenientes de la utilización del PC: Los usuarios de PC encuestados manifiestan muchas más ventajas que inconvenientes en la utilización del PC.

Ventajas de la utilización del PC: Entre las respuestas opcionales, las ventajas más elegidas son (vid. Tabla 3): simplifica la realización de trabajos; simplifica el acceso a Internet y a cualquier tipo de información; tiene otras muchas posibilidades para fines diferentes al estudio; simplifica/posibilita la búsqueda de documentación y la comunicación con la FernUniversität. Las diferencias son escasas entre los grupos según su tipo de discapacidad.

A la pregunta abierta sobre las ventajas en relación con el tipo concreto de discapacidad, se citan entre otras: el PC como medio para compensar problemas y déficits causados por la discapacidad; el PC como ayuda visual; la utilización de e-mail en lugar del teléfono para los deficientes auditivos; PC e Internet como medio para superar una movilidad limitada (p.ej. para usuarios de sillas de ruedas) - para la búsqueda de documentación o comunicarse con los compañeros; posibilita / simplifica la escritura: algunos encuestados con la motricidad de los brazos limitada refieren que no pueden escribir con la mano o que lo hacen con gran dificultad. 
Tabla 3: Ventajas del uso del PC - en opinión de los usuarios del PC

f: frecuencia; \%B: porcentaje, referido a 75 usuarios de PC entre los encuestados; $\% \mathrm{Ng}$ : porcentaje, referido al total de 413 respuestas

\begin{tabular}{|c|c|c|c|}
\hline 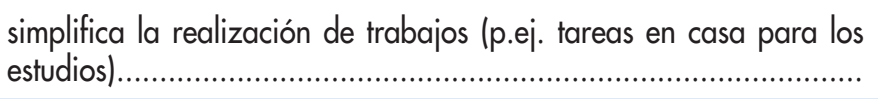 & 67 & 89,3 & 16,2 \\
\hline 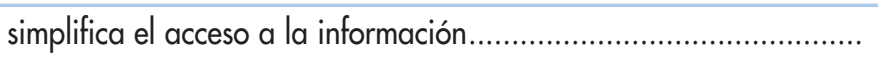 & 54 & 72,0 & 13,1 \\
\hline 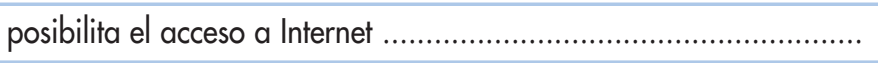 & 58 & 77,3 & 14,0 \\
\hline simplifica / posibilita la comunicación con la Fernuniversität ............. & 45 & 60,0 & 10,9 \\
\hline 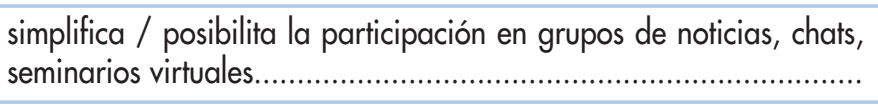 & 27 & 36,0 & 6,5 \\
\hline 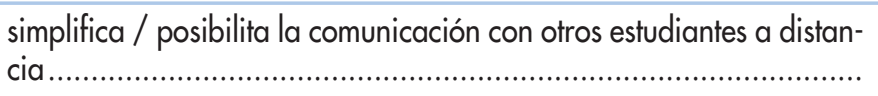 & 27 & 36,0 & 6,5 \\
\hline simplifica / posibilita la búsqueda de documentación ....................... & 47 & 62,7 & 11,4 \\
\hline 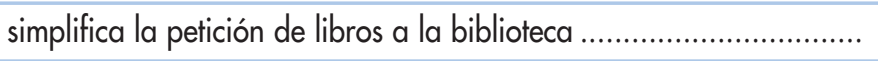 & 31 & 41,3 & 7,5 \\
\hline 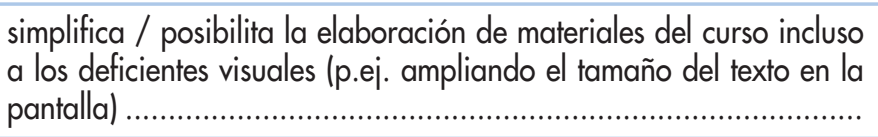 & 7 & 9,3 & 1,7 \\
\hline 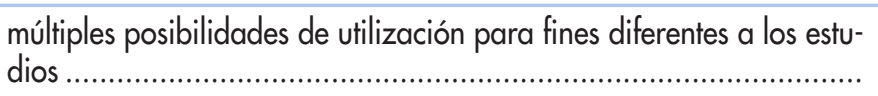 & 50 & 66,7 & 12,1 \\
\hline
\end{tabular}

Inconvenientes de la utilización del PC: Los inconvenientes más señalados por los usuarios de PC encuestados se refieren (vid. Tabla 4) a su coste (de transferencia de datos, pero también de adquisición), los problemas derivados de permanecer sentado frente al aparato y el sobreesfuerzo de la vista y las articulaciones de la mano. Algunos citan las escasas posibilidades de aprendizaje y que las páginas web sobre ofertas multimedia no tienen en cuenta las necesidades especiales de los discapacitados. En los distintos subgrupos hay muchas coincidencias, pero también diferencias muy claras: entre los que padecen «deficiencias del aparato locomotor» hay más respuestas que en los demás sobre los problemas derivados de permanecer frente al PC, mientras que los deficientes visuales señalan más la «sobrecarga y cansancio de la vista» así como la «que la configuración de las páginas web no tiene en cuenta las necesidades de los discapacitados».

En la pregunta abierta sobre otros inconvenientes se indicaron: el aislamiento en el aprendizaje y los altos costes (por la lentitud en la transferencia de datos). Respecto a Internet, se destaca la escasez de información sobre grupos de noticias, chats y seminarios virtuales. Junto a estos inconvenientes generales se citan algunos específicos de cada tipo de discapacidad - como el coste del 
equipamiento especial para discapacitados; las consultas telefónicas sobre los problemas que se les planteen (p.ej. al intentar la conexión con la FernUniversität; esto no sólo sería caro, sino que sería un grave problema para personas con discapacidad auditiva severa); la postura forzada de la cabeza o el cuerpo (que de prolongarse puede ocasionar fuertes dolores); problemas para las personas con graves deficiencias sensoriales visuales o auditivas pues la información multimedia suele ser acústica o visual.

Tabla 4: Inconvenientes de la utilización del PC - en opinión de los usuarios de PC

$f:$ frecuencia; \%B: porcentaje, referido a 75 usuarios de PC entre los encuestados; \% Ng1: porcentaje, referido al número total de 153 respuestas- considerando por separado las subcategorías de costes; \% Ng2: porcentaje, referido al número total de 135 respuestas - con las subcategorías de costes

\begin{tabular}{|c|c|c|c|c|}
\hline manejo demasiado complicado. & 1 & 1,3 & 0,7 & 0,7 \\
\hline $\begin{array}{l}\text { escasas posibilidades de formación para el aprendizaje } \\
\text { del manejo del PC }\end{array}$ & 14 & 18,7 & 9,2 & 10,4 \\
\hline $\begin{array}{l}\text { altos costes (respuestas de una o más de las tres catego- } \\
\text { rías siguientes): }\end{array}$ & 33 & 44,0 & - & 24,4 \\
\hline $\begin{array}{l}\text { - para adquirir el equipo básico (hardware + progra- } \\
\text { mas). }\end{array}$ & 20 & 26,7 & 13,1 & - \\
\hline $\begin{array}{l}\text { - para adquirir el equipo auxiliar necesario (especial } \\
\text { para discapacitados). }\end{array}$ & 3 & 4,0 & 2,0 & - \\
\hline $\begin{array}{l}\text { - de la transferencia de datos / comunicación (p.ej. } \\
\text { costes del teléfono por el uso de Internet)......................... }\end{array}$ & 28 & 37,3 & 18,3 & - \\
\hline problemas en la financiación del equipo auxiliar .............. & 4 & 5,3 & 2,6 & 3,0 \\
\hline $\begin{array}{l}\text { escasa asistencia en el momento de la elección / con- } \\
\text { formación del equipo ideal para mi forma especial de } \\
\text { discapacidad............ }\end{array}$ & 2 & 2,7 & 1,3 & 1,5 \\
\hline $\begin{array}{l}\text { cansancio / agotamiento / problemas posturales tras } \\
\text { periodos prolongados frente al PC..................................... }\end{array}$ & 32 & 42,7 & 20,9 & 23,7 \\
\hline $\begin{array}{l}\text { sobrecarga de las articulaciones de la mano tras un uso } \\
\text { prolongado del PC................................................ }\end{array}$ & 18 & 24,0 & 11,8 & 13,3 \\
\hline cansancio / agotamiento / sobrecarga de la vista........... & 28 & 37,3 & 18,3 & 20,7 \\
\hline $\begin{array}{l}\text { configuración de páginas web o de ofertas multimedia } \\
\text { en Internet que no tiene en cuenta las necesidades de los } \\
\text { discapacitados (p.ej. utilización de elementos gráficos o } \\
\text { de voz sin la alternativa de líneas de texto explicativas).. }\end{array}$ & 3 & 4,0 & 2,0 & 2,2 \\
\hline
\end{tabular}


Sugerencias y propuestas de mejora de los encuestados

Al final, en dos preguntas abiertas, se pide a los encuestados sus sugerencias y propuestas de mejora. Aunque en general ven más ventajas que inconvenientes del PC para su educación a distancia, plantean una serie de propuestas que no se refieren únicamente a la utilización del PC ni a su especial situación como estudiantes discapacitados; muchas de las sugerencias podrían haber sido planteadas por estudiantes no discapacitados. En la siguiente exposición se incide sobre aquellas referidas a la utilización del PC y los medios por los estudiantes discapacitados. Figuran en forma de tabla y son objeto de un breve comentario posterior.

Sugerencias sobre el sistema de estudio en general (en particular sobre discapacidades)

a) Acceso sin barreras y equipamiento adaptado a los discapacitados de los centros de estudio.

b) Elección del lugar de las clases y actos académicos, accesibles con silla de ruedas; información sobre los lugares accesibles para discapacitados físicos y deficientes visuales.

c) Mejora de las posibilidades de comunicación en general para discapacitados; listas regionales de contactos para facilitar la organización de grupos de autoayuda; establecimiento de grupos de noticias especiales para discapacitados.

d) Reducción en las tasas académicas para discapacitados.

sobre a) + b): En la actualidad no todos los centros de estudio permiten el fácil acceso de discapacitados (en particular de usuarios de silla de ruedas) ni están adaptados a sus necesidades (conforme a la norma DIN 18024, Parte 2). No depende sólo de la FernUniversität decidir sobre la realización y el plazo de estas eventuales mejoras, pues además de la necesaria financiación, los centros de estudio por regla general se establecen en cooperación con otras entidades (comunidades, instituciones, etc.).

Sin embargo, no supondría un gasto excesivo el ofrecer información más detallada sobre la accesibilidad de los centros de estudio en la Guía del curso, en la correspondiente página web de la FernUniversität. En la Guía (del curso 1999/2000) en algunos centros de estudio se indica «adecuado para usuarios de silla de ruedas». Esta formulación no aclara si el resto de los centros de estudio son accesibles para usuarios de sillas de ruedas o en qué medida lo son.

Los lugares/locales seleccionados para la celebración de los actos de la FernUniversität (clases presenciales, seminarios, etc.) no son siempre accesi bles para usuarios de sillas de ruedas. Habría que procurar que en el futuro se seleccionaran teniendo más en cuenta su accesibilidad o que al menos en las convocatorias de los actos se informara siempre sobre su (no) accesibilidad. 
Opiniones de estudiantes discapacitados sobre la utilización del ordenador en la educación a distancia

sobre c): La formación de grupos de contacto regionales sobre la base de listas de estudiantes discapacitados podría resultar de gran utilidad para los afectados en muchos aspectos.

Sin embargo, la FernUniversität no puede facilitar estas listas de estudiantes discapacitados, pues la característica «discapacitado/enfermo crónico», (ya) no se consta en la matriculación, entre otras causas por la aplicación de las normas sobre protección de datos. Y la Comisión especial para estudiantes discapacitados, sin una autorización previa, no puede facilitar a terceros los nombres y direcciones de las personas que voluntariamente se registren como discapacitados. Por tanto sería mejor elaborar y facilitar las listas regionales de estudiantes discapacitados a través de los grupos de autoayuda de estudiantes a distancia discapacitados y enfermos crónicos (TANDEM ). Más adelante se aborda la creación de grupos de noticias para estudiantes discapacitados (en las sugerencias sobre la oferta de Internet).

sobre d): En la actualidad la FernUniversität no ofrece reducción o exención en las tasas para ciertos grupos. Los estudiantes con escasos ingresos pueden presentar la correspondiente solicitud y obtener si es el caso la reducción o la exención de las tasas; esta «cláusula social» se refiere a casos particulares, no está destinada a un grupo determinado (como p.ej. discapacitados), y de ella pueden beneficiarse personas de diferentes grupos.

Sugerencias sobre los exámenes:

a) Permiso para utilizar el PC en el examen, en particular a aquellos que debido a su discapacidad no pueden apenas escribir a mano;

b) Más posibilidades para discapacitados con movilidad limitada de hacer los exámenes y pruebas en casa.

c) Superior evaluación general de los trabajos de los discapacitados.

d) Posibilidad de enviar tareas por e-mail o Internet (para evitar ir a correos o al buzón).

sobre a) y b): La Deutsches Studentenwerk (DSW) indica la obligación de las escuelas de compensar a los estudiantes discapacitados sus inconvenientes durante sus estudios y los exámenes ${ }^{34}$. Según una recomendación del Comité de asesoramiento de estudiantes discapacitados de la DSW, en los reglamentos docentes de las universidades alemanas debería constar la formulación siguiente o una similar: «Si mediante un certificado médico un candidato demuestra que, debido a un perjuicio físico de larga duración o una discapacidad, no está en condiciones de realizar los exámenes total o parcialmente en la forma prevista, el presidente del Tribunal deberá conceder al candidato más tiempo para realizar

${ }^{34}$ Deutsches Studentenwerk 1998, pág. 174 y s. 
Ios exámenes o permitir que realice pruebas similares de otra forma. Lo mismo es aplicable al resto de las pruebas durante los estudios. $\rangle^{35}$

En la FernUniversität siempre se ha procurado cumplir esta obligación de compensar los inconvenientes tratando cada caso en particular (por ejemplo, realizando pruebas o exámenes en casa en el caso de discapacitados ${ }^{36}$; o modificando las condiciones del examen en función de la discapacidad particular del examinando). En general suelen encontrarse métodos satisfactorios, pero a veces surgen problemas. Algunos encuestados se quejan de falta de comprensión por parte de algunos examinadores ante las dificultadas derivadas de su discapacidad. Esta se manifiesta entre otras en la prohibición del uso del PC en exámenes, que plantea un verdadero problema a los discapacitados que no pueden escribir o solo pueden hacerlo con un gran esfuerzo. Por el contrario, la DSW recomienda explícitamente que se permitan los medios técnicos necesarios, incluido el ordenador. $^{37}$

sobre c): La «compensación de inconvenientes» que recomienda la DSW no debe interpretarse en el sentido de que el examen del estudiante discapacitado deba ser mejor evaluado en general. La compensación de inconvenientes se refiere a las condiciones que hay que facilitar en función de la discapacidad individual, a fin de que el estudiante pueda examinarse, pero no a los criterios de evaluación del examen.

sobre d): Es muy probable que la FernUniversität disponga en breve de nuevas formas de envío de tareas en función de cada curso. Con el desarrollo de la «universidad virtual» se impondrá la comunicación electrónica (envío de soluciones por e-mail o formularios de respuesta en páginas web).

Propuestas de asesoramiento sobre el uso de hardware y software:

a) Más ayuda para el uso de hardware y soffware; realización de seminarios sobre PC e lnternet especialmente destinados a discapacitados (p.ej. en jornadas de estudiantes discapacitados); en el caso de discapacidad grave o falta de movilidad: enseñanza a domicilio.

b) Información sobre programas auxiliares específicos para discapacitados (hardware y soffware) y asesoramiento para su adquisición e instalación; consejos sobre financiación, adquisición centralizada de estos programas por parte de la FernUniversität.

sobrea): El departamento contable de la0 Universidad está muy preocupado por responder a todas las peticiones de información sobre asistencia y enseñanza

${ }^{35}$ Deutsches Studentenwerk 1998, pág. 175.

${ }^{36}$ Los exámenes además podrían hacerse por videoconferencia, con el profesor en la Central de Hagen y el examinando en un centro de estudio, para evitarle al estudiante discapacitado con movilidad limitada el largo viaje a Hagen.

${ }^{37}$ DSW 1998, pág. 178 
Opiniones de estudiantes discapacitados sobre la utilización del ordenador en la educación a distancia

mediante folletos, ofertas en la red y una hotline, aunque por el momento no se ofrece asesoramiento específico para discapacitados. La realización de seminarios en las jornadas AStA de discapacitados sería de gran ayuda, pues podrían abordarse las necesidades de los usuarios discapacitados de forma específica.

sobre b): Dada la creciente importancia del PC en la educación a distancia, sería deseable que la FernUniversität ofreciera amplia información sobre programas auxiliares destinados a discapacitados o al menos de una serie de enlaces a determinadas páginas web de asociaciones de discapacitados y otras instituciones. En lo que respecta a la adquisición centralizada de este tipo de programas, se plantea la dificultad de determinar las necesidades; además, mantener gran cantidad de estos programas no es muy recomendable, dada la velocidad con la que evoluciona la tecnología en este campo.

Sugerencias sobre la oferta de medios y configuración mediática:

a) Evitar que la oferta informativa sea accesible sólo de forma acústica o visual (p.ej. las ofertas multimedia); configuración que permita el acceso de deficientes visuales y auditivos.

b) Para deficientes visuales: más material docente accesible de forma acústica; si es posible, material de estudio en forma de audiocasete.

c) Mayor esfuerzo en la preparación de material gráfico para deficientes visuales; las fórmulas y figuras deberían tener una descripción verbal.

d) Disponibilidad de los textos de todos los cursos en disquete, CD-ROM o para su descarga desde Internet (de gran ayuda para deficientes visuales, que sólo pueden leer los textos en pantalla después de ampliarlos).

sobrea), b) y c): En estos 3 aspectos se trata del acceso a la oferta mediática de personas con diferentes deficiencias sensoriales.

La cuestión de la accesibilidad, como ya dijimos al referirnos a los usuarios de sillas de ruedas, se refiere a la configuración de entornos de forma que puedan ser utilizados sin mayor problema y en la medida de lo posible por todo el mundo, y en particular por las personas con discapacidades. Esta idea constituye también la base para la configuración orientada a los discapacitados, como por ejemplo las normas sobre configuración de vías públicas y viviendas adaptadas a los discapacitados (DIN 18024 y 18025).

En este sentido, suele suceder que una configuración concebida en principio para responder a las necesidades de un cierto grupo de usuarios, p.ej. para personas con una determinada discapacidad, suele acabar redundando en beneficio de otras personas que no la padecen. El ejemplo más común es el de la bajada de los bordillos en las aceras, que no beneficia sólo a los usuarios de sillas de ruedas, sino también a las madres que llevan de paseo a sus hijos en el cochecito o a los que tiran del carro de la compra. 
También en la configuración de software, interfaces de usuario, ofertas multimedia o páginas web se plantean cuestiones de accesibilidad, normalmente con palabras clave como accessibility o «diseño para todos». La idea es configurar el producto de forma que pueda ser utilizado en la medida de lo posible por todos los usuarios potenciales ${ }^{38}$. Hay ejemplos de ideas que aunque en principio estaban destinadas a usuarios con determinadas necesidades o discapacidades, han servido para otros usuarios. Según Bergman \& Johnson resulta simplista plantear la dicotomía «discapacitado / no-discapacitado», pues entre los usuarios - sean «discapacitados» 0 «no discapacitados» - hay muchos factores que les diferencian en las habilidades necesarias para utilizar estos medios; la mayoría de las personas además, al hacerse mayores, tienen más limitaciones (como hipermetropía, disminución de la movilidad o de la capacidad auditiva); las personas jóvenes, como consecuencia de un accidente o enfermedad, también pueden sufrir provisionalmente graves deficiencias en su capacidad. ${ }^{39}$

La posibilidad de dar órdenes al programa de diferentes formas, no sólo resulta de ayuda para las personas con discapacidades, pues proporciona mayor comodidad a todos los usuarios, en función de sus preferencias o habilidades. La utilización de un tamaño de letra mayor en las páginas web puede que parezca destinado a los deficientes visuales; pero a la vez es de gran ayuda para personas que por su edad sufren de hipermetropía. La inclusión de gráficos en las ofertas multimedia con un texto aclaratorio puede que también parezca pensado para los deficientes visuales, pero a la vez contribuye a la mejor comprensión de estos mensajes por parte de todos los usuarios. El abordar varias modalidades sensoriales, como la posibilidad de elección entre información visual y auditiva en las ofertas multimedia, no sólo facilita el acceso de las personas con deficiencias visuales, sino que constituye un enriquecimiento de la oferta en su conjunto.

En este sentido, Schulmeister aconseja en relación con la utilización de modos visuales y auditivos en las ofertas multimedia: «Ofrecer el mayor número

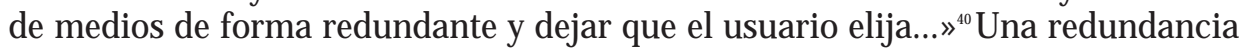
así podría aplicarse también como medio de adaptación de diferentes usuarios, en particular para la ayuda a discapacitados. ${ }^{41}$

sobre d): En la actualidad se dispone de una amplia serie de cursos para deficientes visuales en diversas formas mediáticas (escritura de puntos, casetes,

${ }^{38}$ Una interesante introducción sobre 'accessibility' en la interacción persona-ordenador según las necesidades de los discapacitados es la que dan Bergman \& J ohnson 1995; vid. además Bühler 1998 y los trabajos en Bühler \& Knops 1999 (e.o. el artículo de Emiliani \& Stephanidis 1999).

${ }^{39}$ Bergmann \& J ohnson citan en este sentido una frase de McMillan (1992, pág. 144): "Desde el punto de vista del ordenador, todos los usuarios humanos son discapacitados."

40 Schulmeister 1997, pág. 57, que cita a Bork 1992

${ }^{41}$ Schulmeister 1997, pág. 57, que cita a Edwards 1992. Schulmeister (1997, pág. 57) opina que la cuestión sobre las ventajas e inconvenientes relativos de los medios visuales y auditivos no puede abordarse de forma generalizada. 
Opiniones de estudiantes discapacitados sobre la utilización del ordenador en la educación a distancia

archivos de texto en estenografía para ciegos, adaptados a displays de líneas de Braille), y la oferta se amplía casi constantemente. Sin embargo, algunos deficientes visuales prefieren los textos del curso en forma de archivos (en disquete/ CD-ROM o para descargar), para escucharlos por un 'screen reader' o - si tienen cierta visión residual - leerlo en la pantalla después de ampliarlo lo necesario. EI problema es que en muchos cursos hay elementos no textuales, como gráficos, fórmulas, etc., que se deben completar o sustituir por descripciones verbales.

Sugerencias sobre Internet:

a) Debería evitarse que el uso de Internet se convirtiera en un requisito para estudiar en la FernUniversität, pues no todos los estudiantes discapacitados quieren o pueden usar Internet.

b) Configuración de las páginas web y ofertas que no excluya a las personas con deficiencias visuales o auditivas. Inclusión de gráficos con texto.

c) Inclusión de enlaces en la página principal de la FernUni a páginas web que contengan información especial para discapacitados, como direcciones en Internet o de e-mail de entidades de interés para discapacitados (como la Comisión especial para estudiantes discapacitados, grupos de autoayuda para discapacitados), páginas con información sobre equipos auxiliares destinados a discapacitados.

d) Creación de grupos de noticias especiales para discapacitados.

e) Más información sobre páginas web de interés.

f) Ampliación de la oferta en la red de la «universidad virtual»: si es posible una oferta global de todas las asignaturas en Internet; más seminarios virtuales; más oferta para la descarga general y particular de bibliografía.

sobrea): En la actualidad las ofertas en la red y multimedia para los estudios en la FernUniversität tienen un carácter complementario y la mayoría de las asignaturas ya no pueden abordarse sin Internet o un PC. Si sigue avanzando la implantación de la «universi dad virtual», puede que llegue a plantearse la cuestión de si podría estudiar sin acceso a Internet. La FernUniversität (al igual que otras instituciones docentes) debería preocupase más de los problemas de exclusión que se plantearían a algunos grupos de personas interesadas en recibir formación.

sobre b): Ya hemos mencionado antes la necesidad de configurar las ofertas multimedia de forma que resulten accesibles al mayor número posible de personas. Nos permitimos indicar algo al respecto: existe un instrumento concebido para comprobar la configuración de páginas web conforme a unos criterios mínimos de accesi bilidad para los discapacitados, un programa desarrollado por el Center for Applied Technology (CAST), Ilamado BOBBY ${ }^{42}$. Los criterios que aplica BOBBY son, entre otros, la existencia de equival encias de texto para todos los elementos no textuales y la disponibilidad de resúmenes (verbales) y transcripciones

${ }^{4}$ en Internet http://www.cast.org/bobby/ 
de gráficos, diagramas y tablas. BOBBY comprueba además la compatibilidad de las páginas web con diferentes buscadores.

sobre c): La FernUniversität ya ofrece en Internet las direcciones de importantes entidades de interés para discapacitados distribuida en varias páginas, aunque debería considerarse la posibilidad de reunir los enlaces correspondientes en una página especialmente destinada a los estudiantes discapacitados. En esta página podrían incluirse enlaces a páginas con información sobre equipos auxiliares para discapacitados.

sobred): El establecimiento de grupos de noticias especiales para estudiantes discapacitados podría ser de gran utilidad y sería preferible que se realizara bajo la responsabilidad de los grupos de autoayuda de los estudiantes a distancia discapacitados y enfermos crónicos. La FernUniversität en sus páginas web podría remitir a estos grupos de noticias y participar con sus aportaciones.

sobree) y f): Ya en la actualidad las páginas web de la FernUniversität contienen mucha información y enlaces con direcciones interesantes. Es previsible que estos enlaces, probablemente a través de las páginas de las distintas áreas docentes - seguirá aumentando en el futuro.

La oferta web y multimedia también aumentará en el futuro. La velocidad depende de la medida en que la FernUniversität logre más medios de promoción (del land, federal o de instituciones europeas).

\section{RESUMEN / CONCLUSIONES}

En general, el panorama obtenido resulta extraordinariamente variado y mezclado: en opinión de una mayoría de los encuestados las ventajas de la utilización del PC e Internet superan en mucho los inconvenientes. A pesar de esta valoración general positiva, los encuestados ven muchas posibilidades de mejora, y algunos incluso observan riesgos o rechazan abiertamente su utilización por los más diversos motivos.

Es de esperar que los responsables de las instituciones docentes no pierdan de vista las especiales necesidades de las personas discapacitadas interesadas en recibir formación y que en el futuro las tengan más en cuenta, pues para muchos discapacitados precisamente el estudio y la participación en la vida académica tiene una importancia muy grande y contribuye de un modo esencial a la realización de su anhelo de lograr una vida plena. 
Opiniones de estudiantes discapacitados sobre la utilización del ordenador en la educación a distancia

\section{REFERENGIAS BIBLIOGRÁFICAS}

AdAM. C. (1993).Parte 2: Zur Lage des Behindertensektors und zu Veränderungen in den Lebenszusammenhängen behinderter Menschen. En: Ministerium für Arbeit, Soziales und Gesundheit des Landes Nordrhein-Westfalen (Hrsg.): Behinderte Menschen in Nordrhein-Westfalen. Wissenschaftliches Gutachten zur Lebenssituation von behinderten Menschen und zur Behindertenpolitik in NRW. Düsseldorf BERGMAN, E. \& J OHNSON, E.: Towards accessible human-computer interaction. En: Nielsen, J. (ed.): Advances in human-computer interaction, volumen 5, pág. 87-113. Norwood, NJ : Ablex Publishing 1995. (También en Internet: http://www.sun.com/access/developers/updt.HCl.advance.html; revisado por última vez el 9.11.00).

BoRK, A.(1992). Learning in the twenty-first century interactive multimedia technology. En: Giardina, M. (ed.): Interactive multimedia learning environments. Human factors and technical considerations on design issues (NATO ASI Series F: Computer and Systems Sciences; 93), págs. 2-18. Berlin/Heidelberg: Springer.

BudDE, H.-G. \& LESZCZENSKY, M. (1990). Behinderteund chronisch Krankeim Studium. Ergebnisse einer Sonderauswertung der 12. Sozialerhebung des Deutschen Studentenwerkes im Sommerse mester 1988. Hochschulplanung 82. Hannover: HIS - Hochschul-Informations-System.

BüHLER, CH. (1998). Hilfestellung - Informationstechnologie für Menschen mit Behinderungen. En: ct 1998, Cuaderno 4, pág. 194-198.

BüHLER, CH. \& KNOPS, H. (eds.) (1999) Assistive technology on the threshold of the new millenium. Assistive Technology Research Series, Vol. 6. Amsterdam: IOS Press.

Bundesministerium für BILDUng Und WISSEnSCHAFt (Ed.) (1993) Studieren mit Behinderungen - Dokumentation der Fachtagung anläßlich des zehnjährigen Bestehens der Beratungsstelle für behinderte Studienbewerber und Studenten des DSW. Bonn: Ministerio Federal de Educación y Ciencia.

CHILD, D. (1993). Materialien zum Studium von Behinderten an der Open University. Milton Keynes: Open University.

Daniel, J. (1996) The mega-universities and the knowledge media - Technology strategies for higher education. London: Kogan Page.

Deutsches Studentenwerk E.V. (Hrsg.) (1993): Studienbedingungen behinderter Studierender an den Hochschulen der Bundesrepublik Deutschland - Ergebnisse einer Umfrage vom Februar 1993. Bonn: Deutsches Studentenwerk.

Deutsches Studentenwerk E.V. (Hrsg.) (1998): Studium und Behinderung. Praktische Tips und Informationen des DSW für Studierende mit Behinderungen und chronischen Erkrankungen. Bonn: Deutsches Studentenwerk.

DıсKоPP, K.-H. (1983) Lehrbuch der systematischen Pädagogik. Düsseldorf: Schwann.

DORSCH, F., HÄCKER, H. \& STAPF, K.-H. (Hrsg.) (1983): Dorsch - Psychologisches Wörterbuch. 11. edición revisada. Bern: Huber.

EdwardS, A.D.N. (1992). Redundancy and Adaptability. En: Edwards, A.D.N. \& Holland, S.: Multimedia interface design in education (NATO ASI Series F: Computer and Systems Sciences; 76), págs. 145-155. Berlin/ Heidel berg: Springer.

EmILIANI, P.L. \& StePhanidIS, C. (1999). Accessing the Information Society. En: C. Bühler \& H. Knops (Eds.): Assistive Technology on the Threshold of the New Millenium. Amsterdam: IOS. págs. 28-33. 
Fandel, G., Bartz, R. \& Nickolmann, F. (eds.) (1996). University level Distance Education in Europe Assessment and Perspectives. Weinheim: Deutscher Studien Verlag.

HAufF, M. (Hrsg.) (1998) media@uni-multimedia? Entwicklung - Gestaltung - Evaluation neuer Medien. Medien in der Wissenschaft, Band 6. Münster: Waxmann.

HeILER, H. (1997) Betroffene entdecken das Internet. Leben und Weg, Magazin körperbehinderter Menschen für selbstbestimmtes Leben, 1997 / 2, abril 97, págs. 17 y ss.

Hofmann, K. (1989). Auswertung und Umfrage unter behinderten Fernstudenten. Hagen: FernUniversität.

Hofmann, K. (1994) Zur Behinderten-Tagung des AStA der FernUniversität vom 11. - 13.02.1994. Hagen: FernUniversität.

HOFMANn, K. \& OMmERBORN, R. (1985). Das Fernstudium - behindertengerecht? Zeitschrift für Hochschuldidaktik, 1985, 9 (1), págs. 25-42.

Hofmann, K. \& OMmerborn, R. (1986). Behinderte im Fernstudium. En: Bundesministerium für Bildung und Wissenschaft (Hrsg.): Behinderte Studenten in der Bundesrepublik, pág. 197-212 Bonn.

Hofmann, K. \& Ommerborn, R. (1997) Studium trotz Behinderung. München: Beck.

Holmberg, B. (1995). Theory and practice of distance education. Second edition. London: Routledge.

Holmberg, B. (1996) The discipline of distance education - character and scope in the 1990s. Epistolodidaktika 1996/1, págs. 5-36.

International Council for Distance Education (ICDE) (ed.) (1997). The new learning environment. A global perspective. Proceedings of the 18th ICDE World Conference. June2-6, 1997. The Pennsylvania State University, University Park, Pennsylvania, USA. (Abstract-Band und $C D$ ).

International Council for Distance Education (ICDE) (ed.) (1999). The new educational frontier: teaching and learning in a networked world. Proceedings of the 19th ICDE World Conference on Open Learning and Distance Education. June 20-24, 1999, Vienna. (CD).

Krahn, H. \& Wedekind, J. (Hrsg.) (2000). Virtueller Campus '99. Heute Experiment - morgen Alltag? Medien in der Wissenschaft Band 9. Münster: Waxmann.

Kramme, M. (1996). Opening/Welcome Adresses. En: Fandel, G., Bartz, R. \& Nickolmann, F. (eds.): University Level Distance Education in Europe Assessment and Perspectives. Proceedings of a Workshop J ointly I nitiated and Organised by FernUniversität and EADTU in Hagen, December 1994. Págs. 13-15. Weinheim: Deutscher Studien Verlag .

Kranig, A. \& Ramm, T. (1990). Das Recht der Alten, Hinterbliebenen und Behinderten. Hagen: FernUniversität.

MCMILLAN, W.W. (1992). Computing for users with special needs and models of computerhuman interaction. Conference on Human Factors in Computing Systems, CHI '92, págs. 143-148. Addison Wesley. (cita Bergman \& J ohnson 1999)

MeISTER, J.-J . (1998) Studienverhalten, Studienbedingungen und Studienorganisation behinderter Studierender. München: Bayerisches Staatsinstitut für Hochschulforschung und Hochschulplanung.

MüLlER, K.E . (1996). Der Krüppel. Ethnologia passionis humanae München: C.H. Beck . 
Opiniones de estudiantes discapacitados sobre la utilización del ordenador en la educación a distancia

MüNCH, J . (1997). Be-hindert - Schicksal, Fakt oder soziales Konstrukt? Zum aktuellen Stand der wissenschaftlichen und politischen Diskussion um den Behindertenbegriff. Neue Praxis (np), Zeitschrift für Sozialarbeit, Sozial pädagogik und Sozial politik, 1997, 97/3, págs. 236-243.

OMmerboRn, R. (1994). Fernstudium für Behinderte- Voraussetzungen, Formen und Möglichkéten. Egelsbach: Hänsel - Hohenhausen.

OMmERBORN, R. (1995). Handicapped students at distance teaching university - ten statements and comments. Epistolodidaktika 1995/2, págs. 50-67.

Ommerborn, R. \& Schuemer, R. (2000). Meinungen behinderter Studierender zur PC-Nutzung im Fernstudium. ZIFF-Papiere 114. Hagen: FernUniversität, Zentrales Institut für Fernstudienforschung (ZIFF) .

Open Universiteit (1992). Ook studeren met een handicap? Heerlen: Open universiteit 1992.

Peters, O. (1976). Die FernUniversität. Das ersteJ ahr. Hagen: v.d. Linnepe.

Pschyrembel - Klinisches Wörterbuch, 256., edición revisada. BerlEn: de Gruyter 1990.

RAU, J. (1976). Die FernUniversität wird angenommen! En: Peters, O. (Hrsg.): Die FernUniversität - Das ersteJ ahr. págs. 23-26. Hagen: v.d. Linnepe.

Scheuermann, F. (Hrsg.) (2000). Campus 2000. Medien in der Wissenschaft, Band 10. Münster: Waxmann.

Schlageter, G., Buhrmann, P. \& Mittrach, S. (1997). Telematics for distance education - The Virtual University System. Telematik für den Fernunterricht - Das System der Virtuellen Universität. Zeitschrift für Hochschuldidaktik, 1997, 21 (2), págs. 8-20.

SCHNITZER, K. (1992): Förderung chronisch kranker Studierender - Bericht über die Ergebnisse der 12. und 13. Sozialerhebung. Referat bei der Fachtagung des Deutschen Studentenwerks (DSW) «Studieren mit Behinderungen», 12.-13.10.1992, Wissenschaftszentrum Bonn 1992. (contenido en: Deutsches Studentenwerk, Beratungsstelle für behinderte Studienbewerber und Studenten, Tips und Informationen Nr. 14, 14.12.1992.)

Schnitzer, K., Isserstedt, W., Kahle, I., Leszczensky, M. \& Schreiber, J. (1992). Das soziale Bild der Studentenschaft in der Bundesrepublik Deutschland. 13. Sozialerhebung des Deutschen Studentenwerks. Schriftenreihe Studien zu Bildung und Wissenschaft, 103; hrsg. vom Bundesminister für Bildung und Wissenschaft. Bad Honnef: Bock.

Schnitzer, K., Isserstedt, W., Schreiber, J. \& Schröder, M. (1996). Das soziale Bild der Studentenschaft in der Bundesrepublik Deutschland. 14. Sozialerhebung des Deutschen Studentenwerks - Zusammenfassung. HIS-Kurzinformation A3/96. Hannover: HochschulInformations-System.

Schulmeister, R. (1997): Grundlagen hypermedialer Lernsysteme Theorie- Didaktik - Design. 2. Auflage. München: Oldenbourg.

SIMON, H. (Hrsg.) (1997) Virtueller Campus. Forschung und Entwicklung für neues Lehren und Lernen. Medien in der Wissenschaft, Band 5. Münster: Waxmann.

vom BRUCH, A.: Behinderte und das Internet - Ein Überblick. http://www.heise.de/tp/deutsch/inhalt/te/ 1354/1.html (1998a; última modificación: 17.11.98; última consulta 9.11.00).

VOM BRUCH, A.: Das Internet ist prinzipiell eine große Errungenschaft. Interview mit Karsten Warnke, Leiter des Fachausschusses für Informationstechnik der Deutschen Blindenund Sehbehindertenverbände und Leiter des Arbeitskreises «Multimedia» des Deutschen Vereins der Blinden und Sehbehinderten in Studium und Beruf (DVBS), und mit dem 
Geschäftsführer des DVBS Andreas Bethke. http://www.heise.de/tp/deutsch/inhalt/ te/1355/1.html (1998b; última modificación: 17.11.98 última consulta 9.11.00).

von Prümmer, Ch. \& Rossié, U. (1996). Ausstattung von Fernstudierenden mit Computern. Ergebnisse einer repräsentativen Befragung von ordentlich Studierenden der FernUniversität im Studienjahr 1995/96. Hagen: FernUniversität, Zentrum für Fernstudienentwicklung (ZFE).

Zondanella, A.: Internet - Eine neue Form der Teilnahme behinderter Menschen an der modernen Informationsgesellschaft. http://members.wbs.net/homepages/a/r/m/arminiog/ handicap.htm (1998; última actualización: 08.07.98; última consulta 9.11.00).

\title{
DIREGCIÓN DEL AUTOR:
}

\author{
Dr. Rainer Ommerborn \\ Dr. Rudolf Schuemer \\ FernUniversität - Gesamthochschule - in Hagen \\ 58084 Hagen \\ Alemania
}

